\title{
Feature selection and weighting by nearest neighbor ensembles
}

\author{
Jan Gertheiss*, Gerhard Tutz \\ Ludwig-Maximilians-Universität München, Akademiestraße 1, 80799 München, Germany
}

\section{A R T I C L E I N F O}

\section{Article history:}

Received 13 February 2009

Received in revised form 8 July 2009

Accepted 16 July 2009

Available online 23 July 2009

\section{Keywords:}

Nearest neighbor methods

Variable selection

Ensemble methods

Classification

\begin{abstract}
A B S T R A C T
In the field of statistical discrimination nearest neighbor methods are a well known, quite simple but successful nonparametric classification tool. If the number of predictors increases, however, predictive power normally deteriorates. In general, if some covariates are assumed to be noise variables, variable selection is a promising approach. The paper's main focus is on the development and evaluation of a nearest neighbor ensemble with implicit variable selection. In contrast to other nearest neighbor approaches we are not primarily interested in classification, but in estimating the (posterior) class probabilities. In simulation studies and for real world data the proposed nearest neighbor ensemble is compared to an extended forward/backward variable selection procedure for nearest neighbor classifiers, and some alternative well established classification tools (that offer probability estimates as well). Despite its simple structure, the proposed method's performance is quite good - especially if relevant covariates can be separated from noise variables. Another advantage of the presented ensemble is the easy identification of interactions that are usually hard to detect. So not simply variable selection but rather some kind of feature selection is performed.
\end{abstract}

(C) 2009 Elsevier B.V. All rights reserved.

\section{Introduction}

The rather old nearest neighbor method [1] is one of the simplest and most intuitive techniques in the field of statistical discrimination. The method is nonparametric and memory based, and frequently used in chemometrics (e.g. by [2,3] or [4]); for an introduction see for example [5]. A new observation with unknown class label is placed into the class of the observation from the training set that is closest to the new observation - with respect to some covariates. Despite (or because of) its simplicity nearest neighbor predictions are often accurate. Cover and Hart [6] showed that (for any number of categories) the probability of error of the (one) nearest neighbor rule is bounded above by twice the Bayes error rate. For results when discrimination is based on $k$ nearest neighbors see [7].

Closeness or similarity, respectively distance $d$ of two observations is derived from a specific metric in the predictor space. For a given training set $T=\left\{\left(y_{i}, x_{i}\right) ; i=1, \ldots, n\right\}$, with $y_{i}$ denoting the class membership and the vector $x_{i}=\left(x_{i 1}, \ldots, x_{i p}\right)^{T}$ representing the predictor values, the nearest neighbor classification of a new observation $(y, x)$ is $\hat{y}=y_{[1]}$, with $\left(x_{[1]}\right.$, $\left.y_{[1]}\right)$ denoting the nearest neighbor in the training set, i.e. $d\left(x, x_{[1]}\right)=$ $\min _{1 \leq i \leq n}\left(d\left(x, x_{i}\right)\right)$. A possible distance measure is the so-called Minkowski distance

$d\left(x_{i}, x_{r}\right)=\left(\sum_{j=1}^{p}\left|x_{i j}-x_{r j}\right|^{q}\right)^{1 / q}$.

\footnotetext{
* Corresponding author.

E-mail addresses: jan.gertheiss@stat.uni-muenchen.de (J. Gertheiss), tutz@stat.uni-muenchen.de (G. Tutz).
}

When the Euclidian metric is used one has the special case of $q=2$. All nearest neighbor methods in the following are based on the Euclidian metric.

In this paper nearest neighbor approaches are applied in a more general way. One aspect of the proposed method is feature selection. Our aim is not only classifying (or estimation) but to find the most influential variables and interaction terms. Simple nearest neighborhood estimates tend to be unstable when noise variables are present. Therefore we aim at selecting relevant covariates or interactions between them, and combine the nearest neighborhood predictions based on single or small groups of predictors. Beyond simple classification, we use nearest neighbor concepts as nonparametric estimators for posterior class probabilities - given classes $g=1, \ldots, G$. If not only the first but the $k$ nearest neighbors of observation $i$ are used for classifying $i$, the relative frequency of predictions in favor of category $g$ among these neighbors can be seen as an estimate of the probability of category $g$. These estimates $\hat{\pi}_{i g}$ can take values $h / k, h\{0, \ldots, k\}$, indicating how many times $g$ is observed among the $k$ nearest neighbors of observation $i$. If neighbors are weighted with respect to their distance to the observation of interest, $\hat{\pi}$ can in principle take all values in $[0,1]$. Weighting neighbors is for example proposed by [8] and further investigated and implemented by [9]. By contrast, in the nearest neighbor ensemble proposed in this article, probability estimates unequal to $h / k, h\{0, \ldots, k\}$, do not result from the weighting of neighbors but from combining and weighting different single nearest neighbor estimates.

The paper is organized as follows. In the next section the proposed nearest neighbor ensemble approach is presented. By means of simulation studies its behavior and performance are extensively studied in Section 3. Finally in Section 4 the introduced technique is visualized for some real world data. 


\section{Nearest neighbor ensembles}

Ensemble methods are a general group of methods. Several classifiers - or learners - are fitted, votes are counted, and final classification is given by the most popular class. Nearest neighbor ensembles have been proposed by some authors. Domeniconi and Yan [10] investigated ensembles of nearest neighbor classifiers based on random subsets of predictors, while performing adaptive sampling. That means variable selection is not completely at random, but a probability distribution is employed in the sampling mechanism. The probability distribution is derived from some kind of relevance measure. Thus, the approach is a two-step procedure, because in a first step all the features' relevance needs to be determined by an adequate technique. Yankov et al. [11] dealt with forecasting time series and proposed ensembles of forecasts from different neighborhoods, namely $k_{1}$-NN and $k_{2}$-NN with small $k_{1}$ and larger $k_{2}$. The name ensemble, however, is misleading, since prediction for a given unit is finally based on either $k_{1}-\mathrm{NN}$ or $k_{2}-\mathrm{NN}$. The question what prediction is more suitable needs to be answered by an adequate classifier. Our goal is to perform a variable selection, i.e. selection in the feature space, using the nearest neighbor methods.

\subsection{Basic concept}

We will start with the simplest case when the learners in the ensemble are nearest neighbor estimates based on single variables. Thus, let $\hat{\pi}_{i g(j)}$ be the nearest neighbor estimate of probability that observation $i$ falls in category $g$, if the distance measure is only based on predictor $x_{j}$. The final estimate $\hat{\pi}_{i g}$ is constructed as an ensemble, i.e. the weighted average

$\hat{\pi}_{i g}=\sum_{j=1}^{p} c_{j} \hat{\pi}_{i g(j)}$, with $c_{j} \geq 0 \forall j$ and $\sum_{j} c_{j}=1$.

We explicitly use weights $c_{j}$ on the variables in order to obtain a selection of relevant predictors. These weights - or coefficients - are unknown and need to be determined in some way. They only have to be positive and sum up to one.

Before presenting our strategy for determining these weights, the question shall be answered if further flexibility is possible. Further flexibility would result from weights depending on category g, i.e.

$\hat{\pi}_{i g}=\sum_{j} c_{g j} \hat{\pi}_{i g(j)}$, with $c_{g j} \geq 0 \forall g, j$ and $\sum_{j} c_{g j}=1 \forall g$.

However, it can be shown that the restriction $c_{1 j}=\ldots=c_{G j}=c_{j}$ is the only possibility to ensure that $\hat{\pi}_{i g} \geq 0 \forall g$ and $\Sigma_{g} \hat{\pi}_{i g}=1$ for all possible future estimations $\left\{\hat{\pi}_{i g(j)}\right\}$ with $\hat{\pi}_{i g(j)} \geq 0 \forall g, j$ and $\Sigma_{g} \hat{\pi}_{i g(j)}=1 \forall j$.

Since it is not known where a new observation $i$ (that is to be classified) will be located in the predictor space, it is unknown which values single estimates $\hat{\pi}_{i g(j)}$ will have. Hence it must be ensured that estimates $\hat{\pi}_{i g}$ sum up to one given any estimate $\left\{\hat{\pi}_{i g(j)}\right\}$. The proof of the statement above is given in the Appendix.

\subsection{Determination of weights}

As mentioned before, in the ensemble formula (1) the single nearest neighbor estimates $\hat{\pi}_{i g(j)}$ are considered as fixed, and weights $c_{j}$ need to be determined. For that purpose we primarily choose a loss function - or score $-L(y, \hat{\Pi})$, which quantifies how well the true class labels $y=\left(y_{1}, \ldots, y_{n}\right)^{T}$ are fitted by probability estimates $(\hat{\Pi})_{i g}=\hat{\pi}_{i g}$, $i=1, \ldots, n, g=1, \ldots, G$. As it is seen from the ensemble formula (1), given all single nearest neighbor estimates $\left\{\hat{\pi}_{i g(j)}\right\}$, the matrix $\hat{\Pi}$, consisting of final estimates $\hat{\pi}_{i g}$, is a function of $c=\left(c_{1}, \ldots, c_{p}\right)^{T}$. So for given training data $T=\left\{\left(y_{i}, x_{i}\right) ; i=1, \ldots, n\right\}$ (and hence given estimates $\left.\hat{\pi}_{i g(j)}\right)$ our strategy is minimizing $L(y, \hat{\Pi})$ over all possible $c$.

\subsubsection{Some (im)possible loss functions}

Before introducing loss functions, let us represent the categorial response $y_{i} \in\{1, \ldots, G\}$ by a $G$-dimensional vector $z_{i}=\left(z_{i 1}, \ldots, z_{i G}\right)^{T}$ of the indicator variables

$z_{i g}=\left\{\begin{array}{lc}1, & \text { if } y_{i}=g \\ 0, & \text { otherwise }\end{array}\right.$

With convention " $0 \cdot \infty=0$ ", a somewhat natural loss function is the logarithmic score

$L(y, \hat{\Pi})=\sum_{i} \sum_{g} z_{i g} \log \left(1 / \hat{\Pi}_{i g}\right)$

which can be derived from the likelihood. For a given $p$-dimensional predictor vector $x_{i}$ a single $z_{i}$ is multinomially distributed with one draw:

$z_{i} \sim \operatorname{Mult}\left(1, \pi_{i}\right)$

with the group probabilities depending on $x_{i}$ and being merged into a vector $\pi_{i}=\left(\pi_{i 1}, \ldots, \pi_{i G}\right)^{T}$. So we have $P\left(y_{i}=g \mid x_{i}\right)=\pi_{i 1}^{z_{11}} \cdot \ldots \cdot \pi_{i G}^{z_{i G}}$. For a single observation $y_{i}$ the negative log likelihood is $-l\left(y_{i}, \pi_{i}\right)=-l\left(z_{i}, \pi_{i}\right)=\Sigma_{g} z_{i g}$ $\log \left(1 / \pi_{i g}\right)$. If a sample of $n$ observations is given, we have $l(y, \Pi)=$ $\sum_{i=1}^{n} \Sigma_{g} z_{i g} \log \left(\pi_{i g}\right)$, with $y$ denoting the $n$-dimensional vector of observed classes. The maximum $l(y, \Pi)=0$ is obtained for $\pi_{i g}=1$, if $z_{i g}=1$, and zero otherwise. A single $r\left(\hat{\pi}_{i}\right)=\Sigma_{g} z_{i g} \log \left(1 / \hat{\pi}_{i g}\right)$ may be seen as the Kullback-Leibler-divergence between $z_{i}$ and $\hat{\pi}_{i}$, also called "minus $\log$ likelihood error" [12].

However, in spite of the close connection to the maximum likelihood principle, the logarithmic scoring rule is not really recommendable, because it is too sensitive with respect to differences between very small probabilities (cf. [13]). In the present case this hypersensitivity may be carried to an extreme. If the number of considered neighbors is only modest and the sample is large, it is quite likely that there is at least one observation $i$ with no neighbor from the same category. Then the estimated probability $\hat{\pi}_{i y_{i}}$ for the correct class $y_{i}$ is zero and the logarithmic loss has value $\infty$. So this loss function is inapplicable in case of nearest neighbor predictions.

The hypersensitivity can be removed via a second order Taylor approximation of the logarithmic score, expanded about $\pi_{i g}=1$, if $z_{i g}=1$, and $\pi_{i g}=0$ otherwise. Since

$\frac{\partial L(y, \Pi)}{\partial \pi_{i g}}=\frac{-z_{i g}}{\pi_{i g}}$, if $z_{i g}=1 ; 0$ otherwise,

and

$\frac{\partial^{2} L(y, \Pi)}{\partial \pi_{i g} \partial \pi_{j k}}=\frac{z_{i g}}{\pi_{i g}^{2}}$, if $z_{i g}=1, i=j, g=k ; 0$ otherwise,

we obtain the loss function

$V(y, \hat{\Pi})=\sum_{i} \sum_{g} z_{i g}\left(\left(1-\hat{\pi}_{i g}\right)+\frac{1}{2}\left(1-\hat{\pi}_{i g}\right)^{2}\right)$.

However, by removing the logarithmic score's hypersensitivity $V$ suffers from a new theoretic disadvantage. By contrast to the logarithmic loss, $V$ is not "incentive compatible" [13]. Incentive incompatibility means that the expected loss $E(V)=\Sigma_{y}^{G}=1 \pi_{y} V\left(y, \hat{\pi}_{y}\right)$ for a new observation $y$ with true class probabilities $\pi_{1}, \ldots, \pi_{G}$ is not minimized by $\hat{\pi}_{\mathrm{g}}=\pi_{\mathrm{g}}, g=1, \ldots, G$. For a two-class response with $g \in\{1,2\}$, for example, $E(V)$ is minimized by $\hat{\pi}_{1}=0$, if $\pi_{1}<1 / 3 ; \hat{\pi}_{1}=3 \pi_{1}-1$, if $1 / 3 \leq \pi_{1} \leq 2 / 3 ; \hat{\pi}_{1}=1$, if $\pi_{1}>2 / 3$. An incentive compatible scoring rule that is not hypersensitive is the pure quadratic loss (see e.g. [13])

$Q(y, \Pi)=\sum_{i} \sum_{g}\left(z_{i g}-\pi_{i g}\right)^{2}$, 
which was first introduced by [14] and is sometimes also called Brier score. If the response has more than two categories, it should be kept in mind that the logarithmic score, or its approximation, only looks at the estimated probability of the actual class $y_{i}$, whereas the quadratic loss $Q$ also takes into account how the estimated probabilities are distributed on the false classes. For fixed estimated probability of the true class the loss is higher, if the true class is faced with a single but strong competitor than in the case when probability mass is equally distributed over the false classes.

\subsubsection{Practical implementation}

For practical minimization of the Brier score the procedure is as follows. For each training observation $i$ we create a matrix $P_{i}$ of single estimates

$\left(P_{i}\right)_{g j}=\hat{\pi}_{i g(j)}$.

These matrices are merged into a big matrix $P=\left(P_{1}^{T}|\ldots| P_{n}^{T}\right)^{T}$. The same is done with dummy vectors $z_{i}$, i.e. we have $z=\left(z_{1}^{T}, \ldots, z_{n}^{T}\right)^{T}$. Now the Brier score as function of $c=\left(c_{1}, \ldots, c_{p}\right)^{T}$ can be written in matrix notation:

$Q(c)=(z-P c)^{T}(z-P c)$.

Hence a quadratic optimization problem with restrictions $c_{j} \geq 0 \forall j$ and $\Sigma_{j} c_{j}=1$ arises. So weights $c_{j}$ can be determined using quadratic programming methods, e.g. the $\mathrm{R}$ add-on packages quadprog [15] or kernlab [16]. An alternative interpretation is in terms of regression: $z$ is regressed (without intercept) on the estimated probabilities forming $P$.

\subsection{Variable selection and interactions}

Variable $x_{j}$ is selected if $c_{j} \neq 0$. There are various ways to obtain predictors with zero weights. When determination of weights is completed, variable selection can, for example, be done via hard thresholding, i.e. coefficients less than a certain threshold $t$, for example $t=0.25 \cdot \max _{j}\left\{c_{j}\right\}$, are set to zero. A coefficient less than $0.25 \cdot \max _{j}\left\{c_{j}\right\}$ means that the corresponding predictor does not even have $25 \%$ of the weight of the 'most important' predictor, resp. estimate. Of course, after eliminating some coefficients, the remaining weights need to be rescaled to sum up to one. Alternatively soft thresholding may be applied. When coefficients - denoted by $\tilde{c}_{j}$ - have been determined, we set $c_{j}=\left(\tilde{c}_{j}-t\right)^{+}$(and rescale); $s^{+}$is the positive part of $s$, i.e. $s^{+}=s$, if $s>0$, and $s^{+}=0$ otherwise. The concept of hard and soft thresholding is for example found in [17]. The threshold $t$ can be seen as a tuning parameter, and should be data dependently chosen. A common procedure for tuning parameter determination is K-fold cross-validation, which is also used in the following. For an introduction to K-fold cross-validation, see for example [18].

If thresholding is used, variables (resp. single estimates) are selected after initial determination of weights. Since coefficients fulfilling $\Sigma_{j} c_{j}=1$ are anyway obtained by scaling, restrictions may be primarily disregarded when determining weights. If restrictions are replaced by $\Sigma_{j}\left|\tilde{c}_{j}\right| \leq s$, a lasso type problem [19] arises. That means, a $L_{1}$ penalty is placed on the $\tilde{c}_{j}$ and the penalized criterion

$Q_{P}(\tilde{c})=(z-P \tilde{c})^{T}(z-P \tilde{c})+\lambda \sum_{j}\left|\tilde{c}_{j}\right|$

is minimized (see e.g. [18]). Lasso typical selection characteristics cause $\tilde{c}_{j}=0$ for some $j$. With rescaling and $c_{j}=\tilde{c}_{j}^{+}$a sensible ensemble results. If lasso estimation is done with additional restriction $\tilde{c}_{j} \geq 0$, the positive lasso [20] is obtained. Nevertheless we prefer estimation without these additional constrains, since computation of the original lasso solution is easier. In addition, variable selection is intended and negative coefficients indicate poor predictive capability of the corresponding term.

Finally - and very importantly - the matrix of predictions $P$ can be augmented by including the interactions of predictors. That means adding all predictions $\hat{\pi}_{i g(j l)}$, resp. $\hat{\pi}_{i g(j l m)}$ based on two or even three predictors $j, l$ and $m$. So the (initial) ensemble may consist of much more than $p$ terms. Obviously this is feasible for small scale problems only, because including interactions, $P$ has $p+\left(\begin{array}{l}p \\ 2\end{array}\right)+\ldots$ columns. However, if variable selection is applied, many terms will be excluded, and the final ensemble will contain only a modest number of estimates.

\section{Simulation studies}

To investigate its behavior the presented nearest neighbor ensemble is studied in simulation studies taken from the literature. Particularly when prediction performance is evaluated, proposed approaches should be compared to some alternative procedures, in order to judge the observed performance. So we first introduce our reference methods.

\subsection{Reference methods}

Two important features of the proposed nearest neighbor ensemble are its selection property and the possible identification of interactions. So the ensemble should be compared to another nearest neighbor approach with suchlike features.

\subsubsection{Nearest neighbor forward/backward variable selection}

The procedure is very simple. We just employ a slightly extended forward variable selection approach based on leaving-one-out (LOO) cross-validation. LOO cross-validation means that the $i$ th observation is set aside and predicted using the rest of the data as learning set. This procedure is iterated over all $i=1, \ldots, n$ observations, and the prediction error is calculated (in our case the Brier score). For an introduction to cross-validation procedures in general, see for example [18].

For categorial outcomes, $p$ (potential) predictors and a given (small) $S$ the algorithm used in this paper is as follows.

Nearest neighbor forward variable selection

Step 1: Select up to $S$ predictors from the $p$ predictors for the nearest neighbor classification by LOO cross-validation and minimizing the Brier score.

Step 2: From the remaining predictors select another set of 1 to $S$ predictors by LOO cross-validation and add these predictors to the already chosen one(s).

Step 3: If there is no improvement in prediction accuracy after Step 2, just take the predictor set from Step 1; else repeat Step 2 until there is no improvement in prediction accuracy anymore.

The tuning parameter $S$ can be seen as the number of simple forward selection steps that are checked in one iteration. Furthermore $S$ can be interpreted as the highest interaction that should be detected. The motivation is that the (marginal) discriminative capability of a certain predictor may be lower or completely absent, if other variables are disregarded. Hence relevant predictors may not be selected by simple forward selection procedures. An illustrating example is given in the next section.

Furthermore the given algorithm may be seen as a slightly modified version of a GPTA $(l, r)[21]$ procedure, i.e. go forward $l$ stages by adding $l$ predictors and go backward $r$ stages by deleting $r$ predictors and repeat this process. For small scale problems this approach gives quite good results (cf. [21]). Above all, however, the extended forward selection is able to detect interactions as pointed out before. So we chose it as a kind of reference procedure for nearest neighbor based variable selection. Wherever forward selection is considered, however, backward selection may be seen as an alternative.

Nearest neighbor backward variable selection

Step 1: Perform nearest neighbor classification based on the entire set of $p$ predictors. 
Step 2: By LOO cross-validation select a set of maximum $S$ predictors to be excluded from the reference data for nearest neighbor classification.

Step 3: If there is no improvement in prediction accuracy after Step 2, just take the original predictor set from Step 1; else exclude further sets of 1 to $S$ predictors until there is no improvement in prediction accuracy anymore.

In analogy to the previous paragraph the tuning parameter $S$ can be seen as the number of simple backward selection steps that are checked in one iteration. In the used implementation backward as well as forward variable selection aim at minimizing the (crossvalidated) Brier score on a given training set.

\subsubsection{Some alternative classification tools}

The methods introduced above are compared to the weighted 5 nearest neighbors as proposed in [9], R package kknn) - an approach that can also be found in [8], a commentary on the famous [1]. Additionally we will report the performance of some other well established classification tools; namely linear discriminant analysis (LDA), CART [22] and Random Forests [23]. For estimation we use R packages MASS [24], rpart [25] and random forest [26], see also [27] for further information.

For estimating ensemble weights the corresponding optimization problem (with restrictions) is solved using the interior point optimizer from the R add-on package kernlab [16]. Single estimates forming the ensemble are standard 3 nearest neighbor estimates. Variable selection is done via hard thresholding with threshold $t$ chosen via 3-fold cross-validation.

\subsection{Two classification problems}

We look at two simulated problems from [18]. There are 10 independent features $x_{j}$, each uniformly distributed on $[0,1]$. The two class $0 / 1$ coded response $y$ is defined as follows:

- as an "easy" problem:

$y=I\left(x_{1}>0.5\right)$, and

- as a "difficult" problem:

$y=I\left(\operatorname{sign}\left(\prod_{j=1}^{3}\left(x_{j}-0.5\right)\right)>0\right)$.

Given the easy problem, dark-gray boxplots in Fig. 1 summarize the results in terms of the quadratic loss (Brier score) for 3 nearest neighbors with forward, resp. backward variable selection $(S=4)$ and the developed nearest neighbor ensemble classification technique over 30 realizations of training $(m=200)$ and test $(n=1000)$ data.

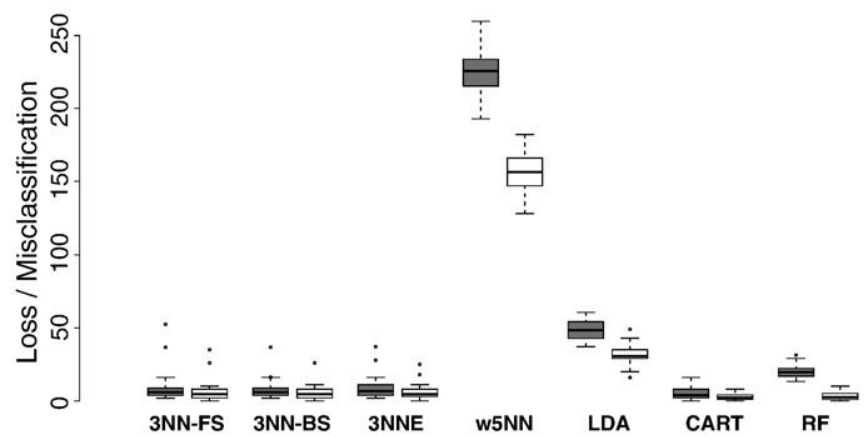

Fig. 1. Boxplots of quadratic loss (dark-gray) and number of misclassified observations (light-colored) over 30 simulations for 3 nearest neighbors with forward/backward variable selection $(S=4), 3$ nearest neighbors ensemble, weighted 5 nearest neighbors, LDA, CART and Random Forests (RF), given the easy classification problem.

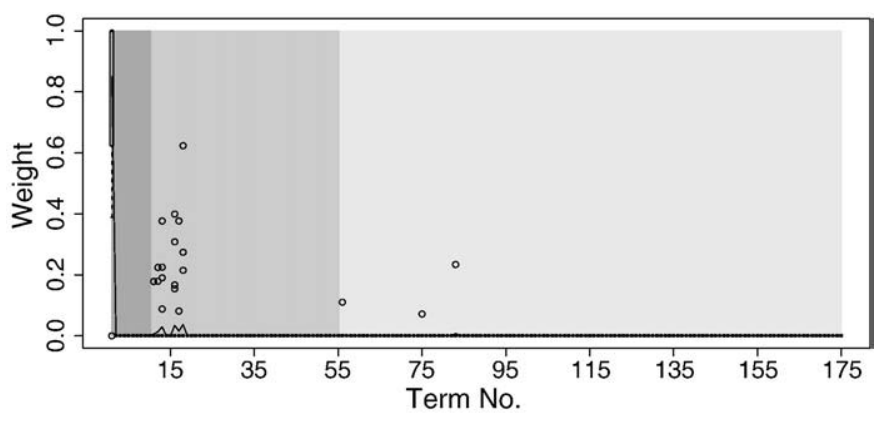

Fig. 2. Boxplots of weights in the nearest neighbor ensemble given the easy classification problem and 30 realizations of training data, each term's average weight is marked by the solid line.

Due to the disadvantage of not being incentive compatible the approximate logarithmic loss is not appropriate for evaluating the performance of the considered methods. The logarithmic score itself is not applicable either, because with just one test observation falling in a category with estimated probability (close to) zero it tends to infinity.

To make variable selection more complicated in the nearest neighbor ensemble, not only predictions based on single predictors are considered but also interaction up to order 3, i.e. predictions using sets of maximum 3 predictors. Since ensemble weights are determined via minimizing the Brier score, the quadratic loss may be seen as a prejudiced measure of prediction accuracy. Hence, as a more neutral quantity, we additionally give the number of misclassified test observations (light-colored), if observations are classified as belonging to the category with highest posterior probability. All considered methods with variable selection properties perform well. By contrast, particularly ordinary nearest neighbor approaches without variable selection get into difficulties caused by noise variables.

In Fig. 2 weights of terms in the nearest neighbor ensemble are shown by means of (degenerated) boxplots. The background indicates estimates based on a single predictor only, estimates using two predictors, and triple interactions respectively. As requested, the nearest neighbor estimate using $x_{1}$ only has the far highest weight in the ensemble. In a few iterations however, also less relevant predictions got some weight.

In Fig. 3 we show the weights of the terms in the ensemble when the difficult classification problem is analyzed. Variable selection is almost perfect. The most important term no. 56 is the prediction based on $x_{1}, x_{2}$ and $x_{3}$. So the good performance (see Fig. 4 ) is not very surprising.

An extended forward selection procedure with $S \geq 3$ can be expected to select the relevant independent variables. But due to the complicated interaction of $x_{1}, x_{2}$ and $x_{3}$, the forward variable selection with $S=1$ would not be successful. We chose $S=4$ again. Also greedy algorithms like CART and the methods based on the latter like Random Forests are not able to identify the right variables. LDA as well is completely inappropriate, and as before, nearest neighbor methods without variable

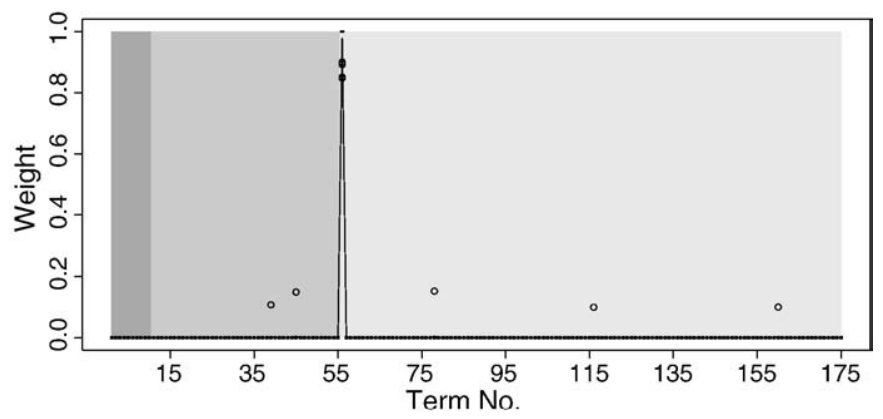

Fig. 3. Boxplots of weights in the nearest neighbor ensemble given the difficult classification problem and 30 realizations of training data, each term's average weight is marked by the solid line. 


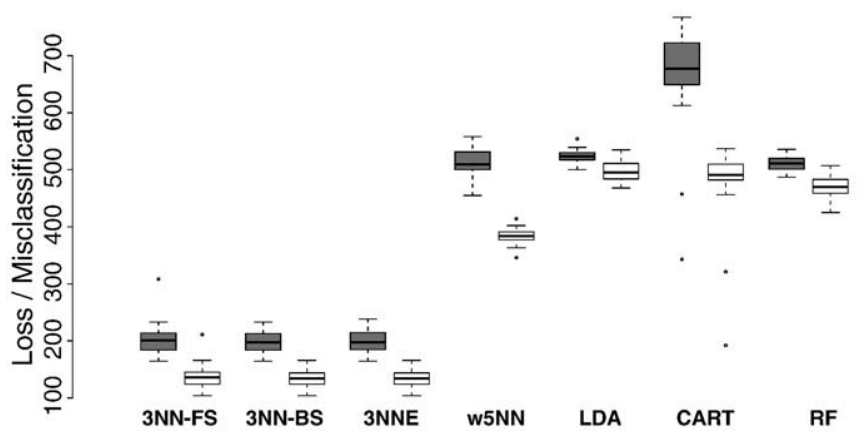

Fig. 4. Boxplots of quadratic loss (dark-gray) and number of misclassified observations (light-colored) for 3 nearest neighbors with forward/backward variable selection $(S=4), 3$ nearest neighbors ensemble, weighted 5 nearest neighbors, LDA, CART and Random Forests (RF), given the difficult classification problem and 30 realizations of training and test set.

selection suffer from the presence of noise variables. In case of backward selection a large $S$ seems to ensure that enough predictors are excluded to outperform nearest neighbor techniques based on the entire set of partly noise - variables. As seen from Fig. 3, when using the ensemble classification the term based on $x_{1}, x_{2}$ and $x_{3}$ has the far highest weight; in many simulation runs it is even the only prediction that is taken into account. So in the difficult classification problem, nearest neighbor ensemble classification performs as well as forward or backward selection.

\section{More examples}

Some further simulation scenarios are taken from [28]. Each training set consists of $m=200$ observations, but our test set size is $n=1000$. Each class has the same number of observations. Exceptions from that rules are indicated. In detail, the following classification problems are investigated, cf. [28]:

1. 2 Dimensional Gaussian: two Gaussian classes in two dimensions $\left(x_{1}, x_{2}\right)$ are separated by 2 units in $x_{1}$. The predictors have variance 1 and 2 , and correlation 0.75 .

2. 2 Dimensional Gaussian with 14 noise: as before, but additionally 14 noise variables having independent standard Gaussian distributions are given.

3. Unstructured: in this example, data with extremely disconnected class structure is simulated. There are 4 classes, each with 3 spherical bivariate normal subclasses, having standard deviation 0.25 . The means of the resulting 12 subclasses are chosen at random (without replacement) from $\{1, \ldots, 5\} \times\{1, \ldots, 5\}$. Each training sample has 20 , each test set 100 observations per subclass.

4. Unstructured with 8 noise: as above, but augmented with 8 independent standard normal predictors.

5. 4 Dimensional spheres with 6 noise: 10 predictors and 2 classes are given. The last 6 predictors are noise variables, with standard Gaussian distributions, independent of each other and the class label. The first 4 predictors in class 1 are independent standard normal, but conditioned on the radius being greater than 3 , whereas the first 4 predictors in class 2 are independent standard normal without restrictions. The first class almost completely surrounds the second class in the 4 dimensional subspace of the first 4 predictors.

6. 10 Dimensional spheres: the situation is similar to the previous example. All 10 predictors in class 1 are independent standard normal, but now conditioned on the squared radius being between 22.4 and 40 , while the predictors in class 2 are again independent standard normal without restrictions. Now there are no pure noise variables. The second class is almost completely surrounded by the first class in the full 10 dimensional feature space.
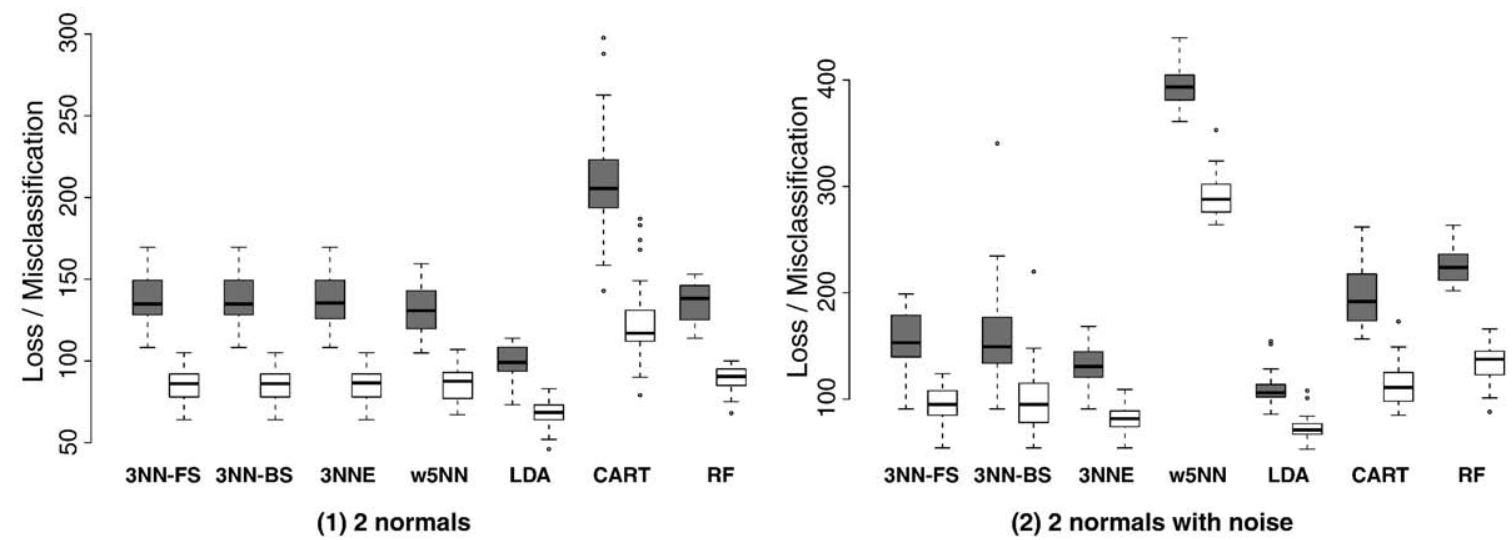

(2) 2 normals with noise
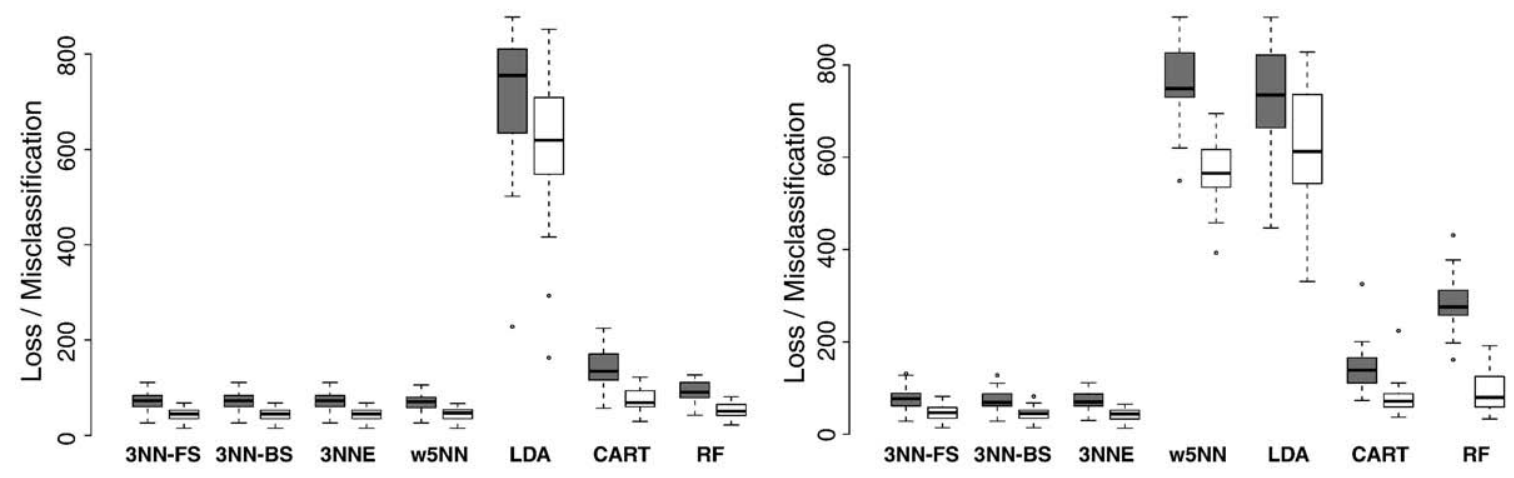

(3) unstructured

(4) unstructured with noise

Fig. 5. Boxplots of quadratic loss (dark-gray) and number of misclassified observations (light-colored) for 3 nearest neighbors with forward/backward variable selection, 3 nearest neighbor ensembles, weighted 5 nearest neighbors, LDA, CART and Random Forests (RF); classification problems 1 to 4. 
7. Constant class probabilities: a 4 class problem is given with class probabilities $(0.1,0.2,0.2,0.5)$, but independent of the predictors. The latter are independent standard normal in 6 dimensions. Here the observed class frequencies may - of course - vary from one realization to another and are far away from being uniformly distributed.

8. Friedman's example: an example like this can be originally found in [29]. There are 2 classes in 10 dimensions. In class 1 the predictors are independent standard normal, in class 2 independent normal with mean and variance proportional to $\sqrt{j}$ and $1 / \sqrt{j}$ respectively, $j=1, \ldots, 10$. That means, all predictors are important, but those with higher index $j$ are more so.

We compare the same classification methods as before, i.e. the proposed nearest neighbor ensemble, nearest neighbor based forward, resp. backward variable selection procedures, weighted 5 nearest neighbors, LDA, CART, and Random Forests. $S=4$ is chosen (if possible) for forward as well as backward selection. In general the highest term in the nearest neighbor ensemble refers to an interaction of order 3 (if possible). To save some computational time in case of scenario 2 we only choose $S=3$ and do not consider triple interactions.

Figs. 5 and 6 show the quadratic loss and the number of misclassified observations from the test set over 30 simulations. Except the first and the last scenario the nearest neighbor ensemble is among the best performing methods. Its performance is particularly high if some noise variables are given. The application of 3-fold cross-validation for the determination of threshold $t$ seems to give good results.

\section{Evaluation of real world data}

When classification tools are visualized and compared this should not be done by simulations only, but also based on real world data sets. For that purpose we use the famous machine learning benchmark glass data set and data from the analysis of Italian olive oils. Before employing our nearest neighbor ensemble (and forward/backward selection) variables are scaled using pooled - or within-class - variances based on the training data. Thus, predictors with one-dimensional discriminative power implicitly obtain some higher weight compared to (widely used) standardizing by overall variances (for details, see [30]). By the R package kknn the latter is done by default.

\subsection{Glass data}

The data can be obtained, for example, from the R add-on package mlbench [31]: 214 observations are given containing examples of the chemical analysis of 6 different types of glass. The problem is to forecast the type of glass on the basis of the chemical analysis. The latter is given in form of 9 metric predictors: (1) refractive index, plus content of (2) sodium, (3) magnesium, (4) aluminum, (5) silicon, (6) potassium, (7) calcium, (8) barium and (9) iron, each measured in weight percent in the corresponding oxide. The possible types of glass are: (I) building windows (float processed), (II) building windows (non-float-processed), (III) vehicle windows, (IV) containers, (V) tableware, (VI) headlamps. The data have also been considered by [23], for example. It has originally been taken from the UCI Repository of Machine Learning Databases [32] at http://www.ics.uci.edu/ $\sim$ mlearn/MLRepository.html. Such kind of study was motivated by criminological investigation; because at the scene of the crime, the glass left can be used as evidence, if it is correctly identified.

For choosing the adequate threshold value we employ 3-fold crossvalidation (Fig. 7, top) and the quadratic loss. If all observations are used for training our nearest neighbor ensemble with hard thresholding and (cross-validation score minimizing) $t=0.2 \max _{j}\left\{c_{j}\right\}$, six terms are selected. The computed weights are seen in Fig. 7 (bottom). All selected terms correspond to triple interactions, but predictors number (8) and (9) are never included. That means barium and iron are not used for classification and may be excluded from discriminant analysis.

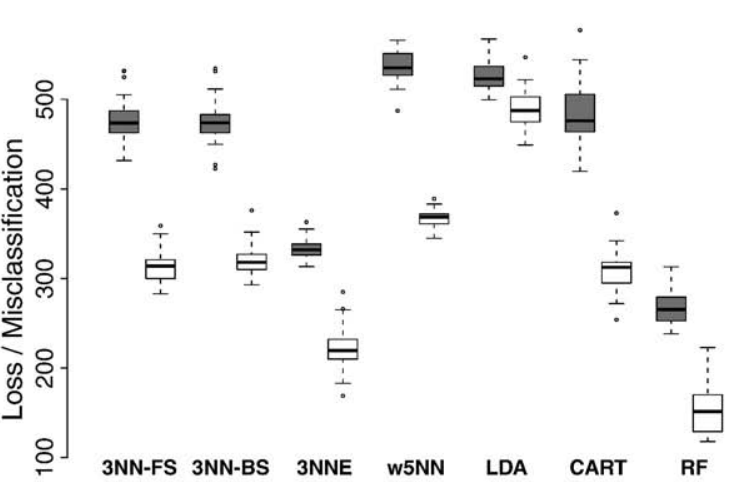

(6) $10 \mathrm{D}$ sphere in 10 dimensions
(5) 4D sphere in 10 dimensions

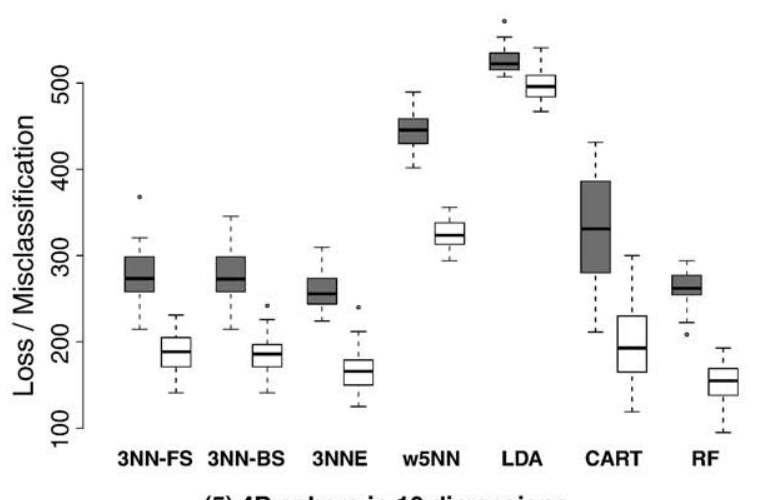

.

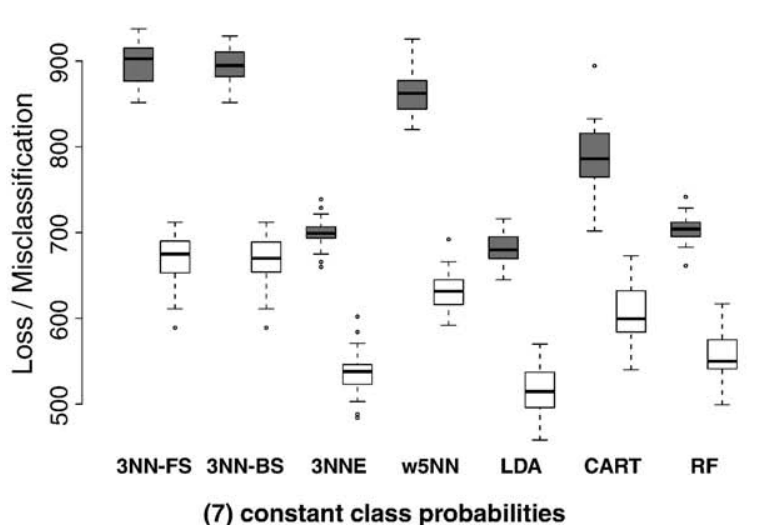

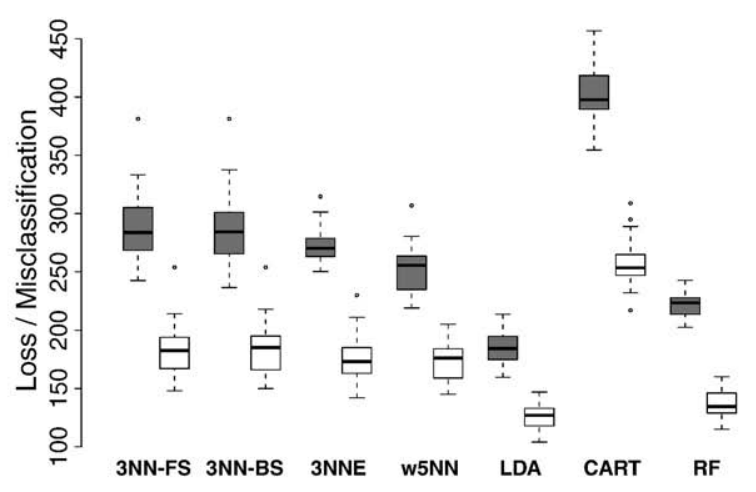

(8) Friedman's example

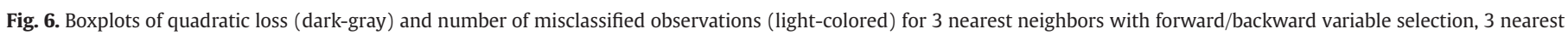
neighbor ensembles, weighted 5 nearest neighbors, LDA, CART and Random Forests (RF); classification problems 5 to 8 . 

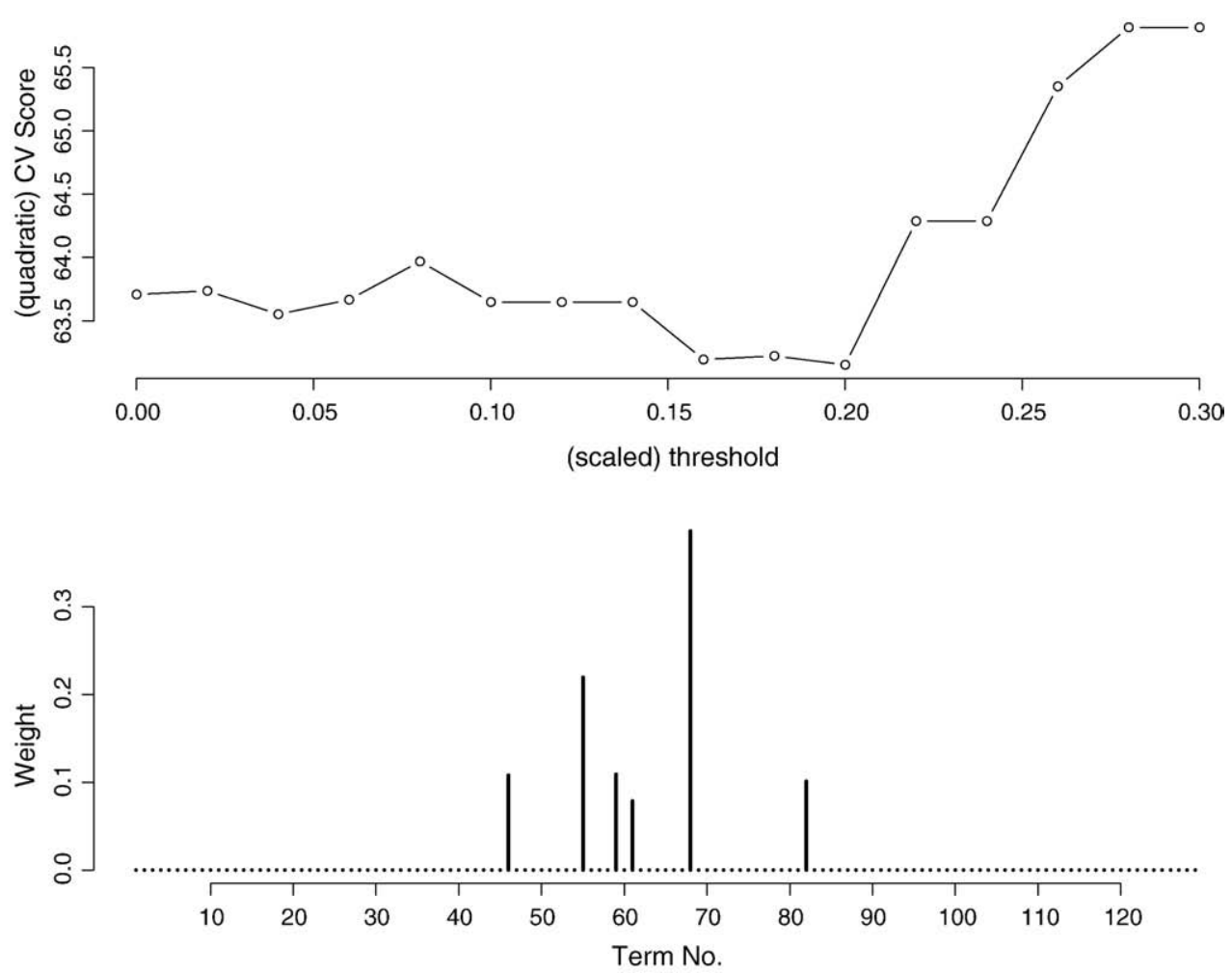

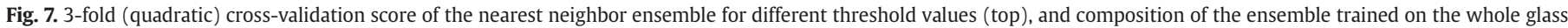
data set and using threshold $t=0.2 \max _{j}\left\{c_{j}\right\}$ (bottom), terms no. 46-129 correspond to triple interactions.

For evaluating the performance of the investigated classification tools (randomly chosen) $20 \%$ of the data are set aside. On the remaining data the methods are trained. The methods are those already compared in the simulation studies above: Nearest neighbor with forward and backward variable selection, nearest neighbor ensembles, weighted 5 nearest neighbors without variable selection, LDA, CART and Random Forests. We have $S=3$, and the highest interaction in the nearest neighbor ensemble is set equal to $S$. The (hard) threshold $t$ is chosen via 3 -fold cross-validation. Not explicitly mentioned tuning parameters have default values. Fig. 8 shows the quadratic loss and number of misclassified observations on the test set over 50 random splits of the data at hand.

If the proposed nearest neighbor ensemble is based on the Brier score it is competitive to Random Forests, which have been shown to perform very well on this data set (see [23]). Furthermore the ensemble is superior to the (weighted) standard nearest neighbor approach. Maybe, because there are some noise variables without discriminative power. For the better performance of the ensemble, however, this is unlikely

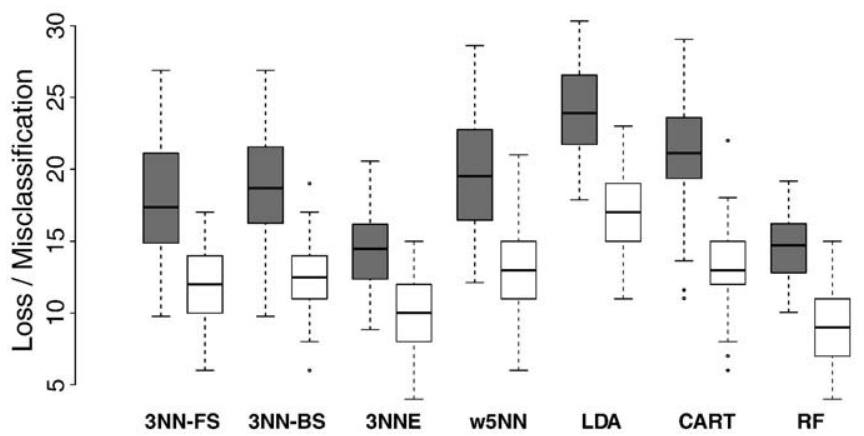

Fig. 8. Boxplots of quadratic loss (dark-gray) and number of misclassified observations (light-colored) for 3 nearest neighbors with forward/backward variable selection, 3 nearest neighbor ensembles, weighted 5 nearest neighbors, LDA, CART and Random Forests (RF), given the glass data and 50 random splits into training and test data. the only reason, since backward or forward selection does not improve nearest neighbor prediction.

\subsection{Olives data}

The data are from [4]. The task is recognizing the geographical origin of Italian olives oils from their fatty acid composition. All in all the different oils are from nine regions, i.e. classes: Calabria, Sicily, Umbria, Coast-Sardinia, Inland-Sardinia, North-Apulia, South-Apulia, East-Liguria, West-Liguria. Only eight predictors (i.e. fatty acids) are given; so in the nearest neighbor ensemble even estimates based on four covariates can be allowed.

Since now more data points (572) than in the previous example are available, (randomly selected) $40 \%$ of the data are used as test set. The same classification tools as before are trained on the remaining data, and serve for discriminant analysis of the test data. As before, this procedure is repeated 50 times. The results in terms of the Brier score (dark-gray) and number of misclassified test observations are shown in Fig. 9. The

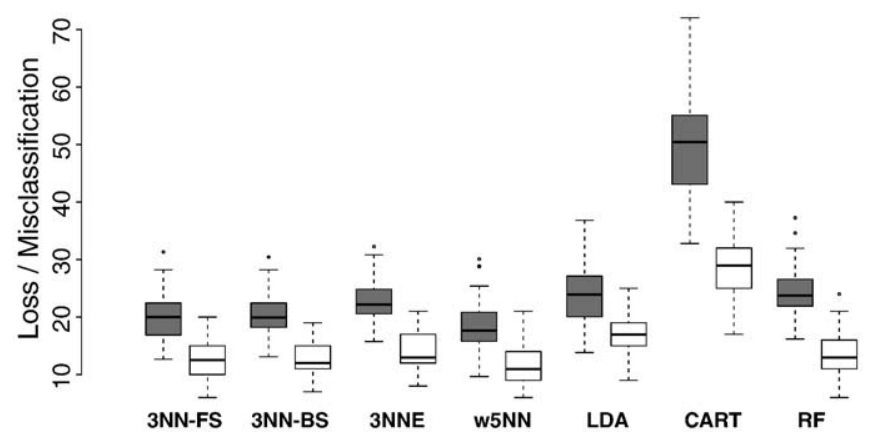

Fig. 9. Boxplots of quadratic loss (dark-gray) and number of misclassified observations (light-colored) for 3 nearest neighbors with forward/backward variable selection, 3 nearest neighbor ensembles, weighted 5 nearest neighbors, LDA, CART and Random Forests (RF), given the olives data and 50 random splits into training and test data. 

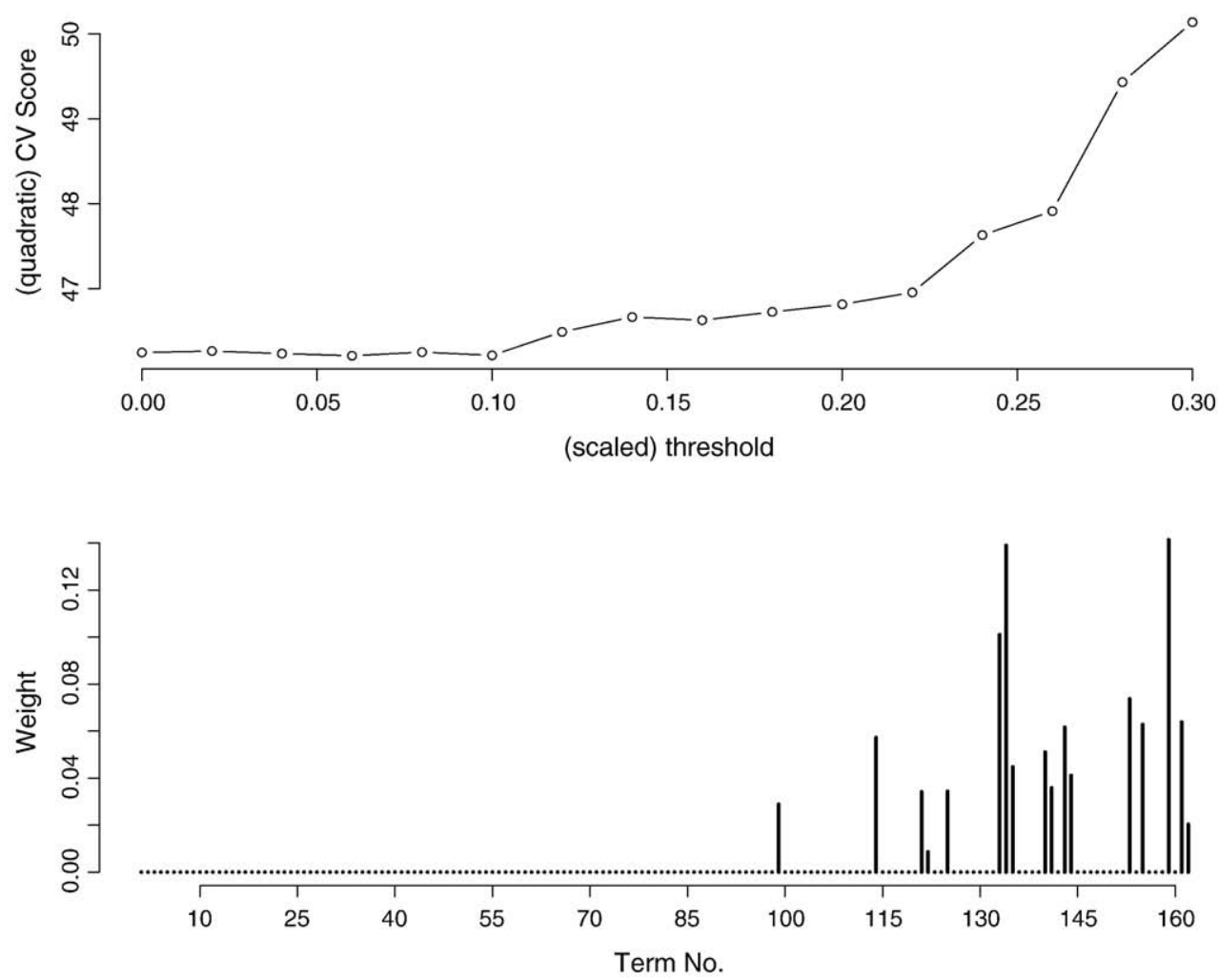

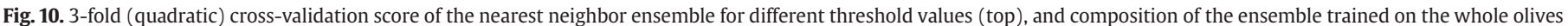
data set and using threshold $t=0.1 \max _{j}\left\{c_{j}\right\}$ (bottom), terms no. 93-162 correspond to subsets of four predictors.

overall winner is the (weighted) standard nearest neighbor method taking into account all predictors at hand. Apparently all predictors have some predictive power. So no improvement can be expected, if some kind of variable selection is applied. Indeed, when the nearest neighbor ensemble is cross-validated and trained on the whole data set (see Fig. 10), a low threshold value is chosen, and only terms of order 4 are selected - covering all 8 predictors.

\section{Summary and discussion}

We propose a nonparametric probability estimation by an ensemble - i.e. weighted average - of nearest neighbor estimates. Each single estimate is based on a single or a very small subset of predictors. In contrast to many other ensemble approaches no randomness is included in the proposed method, e.g. by randomly selecting predictor sets. Instead, all possible predictor combinations up to a previously chosen maximum number of predictors are taken as candidates, and weights are explicitly determined via minimization of a loss function preferably the Brier score. By enforcing many zero weights the final ensemble only consists of a modest number of terms. As a result our ensemble is not a black box (by contrast to many other ensemble methods), but it is directly comprehensible how estimation is carried out. Covariates which are not contained in the selected predictor sets do not serve for classification, or estimation of posterior probabilities. That means variable selection is explicitly done.

The proposed ensemble approach shows good performance for small scale problems, particularly if pure noise variables can be separated from relevant covariates. Easy identification of interactions is another advantage of the presented method. If the largest set of covariates is adequately chosen, even interactions that are usually hard to detect should be identified. So even if classification shall be done by another method, the ensemble may be used for variable selection.

Direct application of the proposed technique to high dimensional problems with interactions, however, is not recommended. If the number of potential predictors is high, interactions cannot be taken into account (because the number becomes too high). But given the microarrays in genetics for example, the presented ensemble might be useful as a nonparametric gene preselection tool. Genes may be ranked according to their weight in the ensemble, and further analysis can be based on the "best genes" only.

Furthermore, ensemble methodology may be applied for automatic choice of the most appropriate metrics, or semi-metrics (along the lines of [33]); or the right neighborhood(s). For that purpose terms in the ensemble should be the nearest neighbor estimates based on different (semi-)metrics or different neighborhoods.

Finally, application to regression problems is possible as well. The concept of minimizing loss functions can be directly adopted, since the quadratic loss is the somewhat natural choice in regression problems.

\section{Acknowledgements}

This work was partially supported by DFG project TU62/4-1 (AOBJ: 548166)

\section{Appendix A}

Proposition 1. Given the following ensemble formula for computing the probability that observation i falls in category g:

$\hat{\pi}_{i g}=\sum_{j} c_{g j} \hat{\pi}_{i g(j)}$, with $c_{g j} \geq 0 \forall g, j$ and $\sum_{j} c_{g j}=1 \forall g$;

restriction $c_{1 j}=\ldots=c_{G j}=c_{j}$ is the only possibility to ensure that $\hat{\pi}_{i g} \geq 0 \forall g$ and $\Sigma_{g} \hat{\pi}_{i g}=1$ for all possible future estimations $\left\{\hat{\pi}_{i g(j)}\right\}$ with $\hat{\pi}_{i g(j)} \geq 0 \forall g, j$ and $\Sigma_{g} \hat{\pi}_{i g(j)}=1 \forall j$.

Proof. We first show that the given restriction has the desired effect. Since $\hat{\pi}_{i g(j)} \geq 0 \forall g, j$, trivially follows $\Sigma_{j} c_{j} \hat{\pi}_{i g(j)} \geq 0$; furthermore

$\sum_{g} \sum_{j} c_{j} \hat{\pi}_{i g(j)}=\sum_{j} c_{j} \sum_{g} \hat{\pi}_{i g(j)}=\sum_{j} c_{j}=1$. 
In the next step we assume that $c_{g j}$ vary over categories $g$ for at least one $j$, i.e. coefficients $c_{g j}$ can be ordered in terms of $c_{[1] j} \leq \ldots \leq c_{[G] j}$ with at least one $\leq$ being a $<$. Now we create groups $J_{r}=\left\{j \mid c_{r j}=\max _{g} c_{g j}\right\}$, $r=1, \ldots, G$. If $\max _{g} c_{g j}$ is not unique for $j$, arbitrarily choose maximum $c_{g j}$ to have disjoint $J_{r}$ but covering all $j$.

Since $\Sigma_{j} c_{g j}=1 \forall g$, there must be at least two $j$ with coefficients differing over categories and $\max _{g} c_{g j}$ in different categories. Hence there are at least two nonempty sets $J_{r_{1}}$ and $J_{r_{2}}$. Let the nonempty sets be denoted by $J_{r_{1}, \ldots .} J_{r_{1}}$ If now $\hat{\pi}_{i r_{r}(j)}=1 \forall j \in J_{r_{t}}$ (which is not completely unlikely in case of nearest neighbor predictions), due to the < above, it follows:

$\sum_{g} \hat{\Pi}_{i g}=\sum_{g} \sum_{j} c_{g j} \hat{\pi}_{i g(j)}=\sum_{j \in J_{r_{1}}} c_{r_{1} j}+\ldots+\sum_{j \in J_{r_{l}}} c_{r_{l} j}>1$.

\section{References}

[1] E. Fix, J.L. Hodges, Discriminatory Analysis - Nonparametric Discrimination: Consistency Properties, US Air Force School of Aviation Medicine, Randolph Field Texas, 1951.

[2] B.M. Lukasiak, S. Zomer, R.G. Brereton, R. Faria, J.C. Duncan, Pattern recognition and feature selection for the discrimination between grades of commercial plastics, Chemometrics and Intelligent Laboratory Systems 87 (2007) 18-25.

[3] R. Japon-Lujan, J. Ruiz-Jimenez, M.D. Luque De Castro, Discrimination and classification of olive tree varieties and cultivation zones by biophenol contents, Journal of Agricultural and Food Chemistry 54 (2006) 9706-9712.

[4] M. Forina, C. Armanino, S. Lanteri, E. Tiscornia, Classifiaction of olive oils from their fatty acid composition, in: H. Martens, H. Russwurm (Eds.), Food Research and Data Analysis, Proceedings from the IUFoST Symposium, Applied Science Publishers LTD, London/New York, 1982, pp. 189-214.

[5] B.R. Kowalski, C.F. Bender, The k-nearest neighbor classification rule (pattern recognition) applied to nuclear magnetic resonance spectral interpretation, Analytical Chemistry 44 (1972) 1405-1411.

[6] T.M. Cover, P.E. Hart, Nearest neighbor pattern classification, IEEE Transactions on Information Theory 13 (1967) 21-27.

[7] B.D. Ripley, Pattern Recognition and Neural Networks, University Press, Cambridge, 1996.

[8] B.W. Silverman, M.C. Jones, Commentary on Fix and Hodges (1951): An important contribution to nonparametric discriminant analysis and density estimation, International Statistical Review 57 (1989) 233-238.

[9] K. Hechenbichler, K. Schliep, Weighted k-nearest-neighbor techniques and ordinal classification, SFB 386 Discussion Paper 399, Ludwig-Maximilians-Universität München, 2004.

[10] C. Domeniconi, B. Yan, Nearest neighbor ensemble, Proceedings of the 17th International Conference on Pattern Recognition. Cambridge, UK, 2004.
[11] D. Yankov, D. DeCoste, E. Keogh, Ensembles of nearest neighbor forecasts, in: J. Fürnkranz, T. Scheffer, M. Spiliopoulou (Eds.), Lecture Notes in Computer Science, Proceedings of the 17th European Conference on Machine Learning, Springer, Berlin/Heidelberg, 2006, pp. 545-556.

[12] S. Le Cessie, J.C. van Houwelingen, Ridge estimators in logistic regression, Applied Statistics 41 (1992) 191-201.

[13] R. Selten, Axiomatic characterization of the quadratic scoring rule, Experimental Economics 1 (1998) 43-62.

[14] G.W. Brier, Verification of forecasts expressed in terms of probability, Monthly Weather Review 78 (1950) 1-3.

[15] B.A. Turlach, quadprog: Functions to solve Quadratic Programming Problems, 2007 $\mathrm{R}$ package version 1.4-11, S original by Berwin A. Turlach, $\mathrm{R}$ port by Andreas Weingessel.

[16] A. Karatzoglou, A. Smola, K. Hornik, A. Zeileis, kernlab - an S4 package for kernel methods in R, Journal of Statistical Software 11 (9) (2004) 1-20.

[17] J. Fan, R. Li, Variable selection via nonconcave penalized likelihood and its oracle properties, Journal of the American Statistical Association 96 (2001) 1348-1360.

[18] T. Hastie, R. Tibshirani, J.H. Friedman, The Elements of Statistical Learning, Springer New York, 2001.

[19] R. Tibshirani, Regression shrinkage and selection via the lasso, Journal of the Royal Statistical Society B 58 (1996) 267-288.

[20] B. Efron, T. Hastie, I. Johnstone, R. Tibshirani, Least angle regression, Annals of Statistics 32 (2004) 407-499.

[21] M. Kudo, J. Sklansky, Comparison of algorithms that selected features for pattern classifiers, Pattern Recognition 33 (2000) 25-41.

[22] L. Breiman, J.H. Friedman, R.A. Olshen, J.C. Stone, Classification and Regression Trees, Wadsworth, Monterey, CA, 1984.

[23] L. Breiman, Random forests, Machine Learning 45 (2001) 5-32.

[24] W.N. Venables, B.D. Ripley, Modern Applied Statistics with S, fourth editionSpringer, New York, 2002.

[25] T.M. Therneau, B. Atkinson, rpart: Recursive Partitioning, 2007 R package version 3.1-36, $\mathrm{R}$ port by Brian Ripley.

[26] A. Liaw, M. Wiener, Classification and regression by random forest, R News 2 (3) (2002) 18-22.

[27] R Development Core Team. R: a Language and Environment for Statistical Computing. R Foundation for Statistical Computing, Vienna, Austria, 2009. ISBN 3-900051-07-0.

[28] T. Hastie, R. Tibshirani, Discriminant adaptive nearest neighbor classification, IEEE Transactions on Pattern Analysis and Machine Intelligence 18 (1996) 607-616.

[29] J.H. Friedman, Flexible metric nearest neighbor classification, Stanford University, Technical report, 1994.

[30] J. Gertheiss, G. Tutz, Variable scaling and nearest neighbor methods, Journal of Chemometrics 23 (2009) 149-151.

[31] F. Leisch, E. Dimitriadou, mlbench: machine learning benchmark problems, $2007 \mathrm{R}$ package version 1.1-3.

[32] D.J. Newman, S. Hettich, C.L. Blake, C.J. Merz, UCI repository of machine learning databases, University of California, Department of Information and Computer Science, Irvine, CA, 1998.

[33] F. Ferraty, P. Vieu, Nonparametric Functional Data Analysis, Springer, New York, 2006. 